\title{
Depressive symptoms and liver fat in subjects with nonalcoholic fatty liver disease after 6-month weight loss intervention: The FLiO study
}

\author{
Bertha Araceli Marin-Alejandre ${ }^{1}$, Itziar Abete ${ }^{1,2}$, Irene Cantero ${ }^{1}$, Cristina Galarregui ${ }^{1}$, \\ Mariana Elorz ${ }^{3,4}$, Josep A. Tur ${ }^{2,5}$, J. Alfredo Martinez ${ }^{1,2}$ and M. Angeles Zulet ${ }^{1,2}$ \\ ${ }^{1}$ Department of Nutrition, Food Sciences and Physiology and Centre for Nutrition Research, Faculty of Pharmacy and \\ Nutrition, University of Navarra, Pamplona, Spain, \\ ${ }^{2}$ Biomedical Research Centre Network in Physiopathology of Obesity and Nutrition (CIBERobn), Instituto de Salud \\ Carlos III, Madrid, Spain, \\ ${ }^{3}$ Navarra Institute for Health Research (IdiSNA), Pamplona, Spain, \\ ${ }^{4}$ Department of Radiology, Clinica Universidad de Navarra, Pamplona, Spain and \\ ${ }^{5}$ Research Group on Community Nutrition and Oxidative Stress, University of Balearic Islands, Palma, Spain
}

\begin{abstract}
Introduction: Nonalcoholic fatty liver disease (NAFLD) is the most common cause of liver disease in Western countries and is strongly associated with obesity and insulin resistance-related comorbidities. Moreover, there is some evidence of a relationship between NAFLD and depression. The aim of this study was to compare the effect of two personalized energy-restricted diets on liver fat and depressive symptoms in overweight or obese subjects with NAFLD after a 6-month follow-up.
\end{abstract}

Materials and methods: Ninety-eight overweight or obese adults with NAFLD were enrolled and randomized to follow one of two different personalized energy-restricted diets (American Heart Association vs. FLiO diet) accompanied by healthy lifestyle advice. Study registered as FLiO: Fatty Liver in Obesity; NCT03183193. Anthropometry, body composition, biochemical features and liver status were assessed at baseline and after a 6-month follow-up. Liver fat was evaluated by Magnetic Resonance Imaging and depressive symptoms using the Beck's Depression Inventory-II (BDI-II).

Results: Participants of both groups showed significant reductions in body weight, total fat mass, glucose, insulin and alanine aminotransferase ( $p<0.001$ for all these parameters in both groups). A significant decrease in liver fat ( $p<0.001$ in both groups) and depressive symptoms ( $p<0.01$ in both groups) was observed after the follow-up. The effects of the intervention in the evaluated variables did not differ when both diets were compared. Consequently, the two groups were considered together as one sample for the further analyses. Correlation analyses evidenced a positive association between the decrease in depressive symptoms and the reduction in body weight $(r=0.241 ; p=0.044)$ and liver fat $(r=0.251 ; p=0.046)$.

Discussion: Previous studies have reported that the prevalence of depression in patients with chronic liver disease (including NAFLD) is higher than in the general population and that major depressive disorder is associated with more severe hepatic steatosis and with worse outcomes in the treatment of NAFLD subjects. In our study, both healthy personalized energy-restricted diets were able to improve metabolic parameters, liver fat content and depressive symptoms in overweight and obese participants with NAFLD. To our knowledge, this is the first study to report an association between the changes in depressive symptoms and the decrease in liver fat after a dietary randomized controlled trial. Further investigation is needed to clarify the relationship between depression and the development and treatment of NAFLD.

\section{Acknowledgments}

Government of Navarra (61/2015), CIBERobn, Fundació La Marató de TV3 (201630.10). B.A.M.-A. is receiving a grant from CONACYT y el Estado de Michoacán (440104/2016).

\section{Conflict of Interest}

"There is no conflict of interest". 